\title{
Compton Scattering by the Nucleon *
}

\author{
Martin Schumacher ${ }^{\text {a }}$ \\ a Zweites Physikalisches Institut der Universität Göttingen, \\ Friedrich-Hund-Platz 1, D-37077 Göttingen, Germany
}

\begin{abstract}
The status of Compton scattering by the nucleon at energies of the first and second resonance is summarized. In addition to a general test of dispersion theories and a precise determination of polarizabilities, the validities of four fundamental sum rules are explored. Recommended averages of experimental values for the electromagnetic polarizabilities and spin-polarizabilities of the nucleon are determined: $\alpha_{p}=12.0 \pm 0.6, \beta_{p}=1.9 \mp 0.6, \alpha_{n}=$ $12.5 \pm 1.7, \beta_{n}=2.7 \mp 1.8\left(\right.$ unit $\left.10^{-4} \mathrm{fm}^{3}\right), \gamma_{\pi}^{(p)}=-38.7 \pm 1.8, \gamma_{\pi}^{(n)}=58.6 \pm 4.0\left(\right.$ unit $\left.10^{-4} \mathrm{fm}^{4}\right)$.
\end{abstract}

Key words: Compton scattering, nucleon, polarizability PACS: $13.60 \mathrm{Fz}, 14.20 \mathrm{Dh}$

\section{Introduction}

One of the central challenges of hadron physics in the regime of strong (non-perturbative) QCD is to identify the relevant degrees of freedom of the nucleon and to quantitatively explain experimental data in terms of these degrees of freedom. Among the processes studied so far Compton scattering plays a prominent role because of the well understood properties of the electromagnetic interaction. Compton scattering is parameterized in terms of generalized polarizabilities of which the invariant amplitudes given in $[1,2]$ are the most convenient ones. Scattering proceeds through

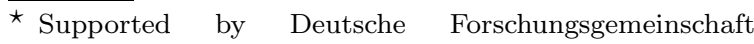
SPP(1034) and projects SCHU222 and 436 RUS 113/510. Presented on P.A. Cherenkov and Modern Physics, Moscow, June 22 - 25, 2004

Email address: mschuma3@gwdg.de (Martin Schumacher).
}

Born terms and through excitation of internal degrees of freedom of the nucleon, the latter leading to the electromagnetic polarizabilities $\alpha$ and $\beta$ and to the spin polarizabilities $\gamma_{0}$ and $\gamma_{\pi}$. These quantities may be understood as low-energy limits of invariant amplitudes [1-3]. Exchanges in the $t$-channel are responsible for the validities of four sum rules, viz. the Baldin or Baldin-Lapidus [4] (BL) sum rule for $(\alpha+\beta)$, the Gerasimov-DrellHearn [5] (GDH) sum rule for $\kappa^{2}(\kappa$ being the anomalous magnetic moment), the BernabeuEricson-FerroFontan-Tarrach [6] (BEFT) sum rule for $(\alpha-\beta)$ and the Lvov-Nathan [7] (LN) sum rule for $\gamma_{\pi}$. A test of these sum rules, therefore, contains valuable information on the physical properties of these exchanges. The $t$-channel exchanges $\pi^{0}$ and $\sigma$ dominate the sizes of $\gamma_{\pi}$ and $(\alpha-\beta)$, respectively, and, therefore, have to be included into the model of the nucleon [8]. 


\section{New Results}

\subsection{Electromagnetic polarizabilities and BL sum} rule

Precise experimental values for the polarizabilities of the proton are available through a recent reevaluation of all low-energy Compton scattering experiments carried out in the 1950's to 1990's [9] and through a recent experiment carried out at MAMI (Mainz) [10]. The results are listed in lines 2 and 3 of Table 1 . The two results for the sum $\alpha+\beta$ of electromagnetic polarizabilities listed in

Table 1

Electromagnetic polarizabilities of the proton.

\begin{tabular}{|c|c|c|c|}
\hline$\alpha_{p}$ & $\beta_{p}$ & $\alpha_{p}+\beta_{p}$ & \\
\hline $11.7 \pm 1.1$ & $2.3 \pm 1.1$ & $14.0 \pm 1.6$ & {$[9]$} \\
$11.9 \pm 1.4$ & $1.2 \pm 0.8$ & $13.1 \pm 1.6$ & {$[10]$} \\
\hline $11.8 \pm 0.9$ & $1.6 \pm 0.6$ & $13.6 \pm 1.1$ & $\mathrm{a})$ \\
$12.0 \pm 0.6$ & $1.9 \mp 0.6$ & $13.9 \pm 0.3$ & $\mathrm{~b})$ \\
\hline
\end{tabular}

a) weighted average of the data in lines 2 and 3

b) the same as a) but constrained by the BL sum rule $[10,11]$

lines 2 and 3 have a weighted average of $\alpha+\beta=$ $13.6 \pm 1.1$ which has to be compared with the BL sum-rule prediction

$$
\alpha+\beta=\frac{1}{2 \pi^{2}} \int_{\omega_{0}}^{\infty} \frac{\sigma_{\text {tot }}(\omega)}{\omega^{2}} d \omega
$$

obtained from photo-absorption data, viz. $13.9 \pm$ $0.3[10,11]$. The excellent agreement of the two results may be considered as a precise confirmation of the BL sum rule.

For the electromagnetic polarizabilities of the neutron three different methods have led to results of good precision. These are the electromagnetic scattering of slow neutrons in the Coulomb field of heavy nuclei [12], the quasi-free Compton scattering on neutrons initially bound in the deuteron [13] and the coherent-elastic scattering of photons by the deuteron [14]. The results are given in Table 2 . Since the model dependence of the method of coherent-elastic scattering is still under discussion we recommend to base the average only on the two other results.
Table 2

Electromagnetic polarizabilities of the neutron determined by three different methods and their recommended average.

\begin{tabular}{|l|l|}
\hline experimental method & polarizability \\
\hline electromagnetic scattering [12] & $\alpha_{n}=12.6 \pm 2.5$ \\
quasi-free Compton scattering [13] & $\alpha_{n}=12.5 \pm 2.3$ \\
coherent-elastic scattering [14] & $\alpha_{n}=8.8 \pm 3.8$ \\
\hline recommended average with $\beta_{n}$ & $\alpha_{n}=12.5 \pm 1.7$ \\
constrained by $\alpha_{n}+\beta_{n}=15.2 \pm 0.5[11]$ & $\beta_{n}=2.7 \mp 1.8$ \\
\hline
\end{tabular}

\subsection{The GDH sum rule}

The integrand of the GDH sum rule

$$
\frac{2 \pi^{2} \alpha_{e} \kappa^{2}}{M^{2}}=\int_{\omega_{0}}^{\infty} \frac{\sigma_{3 / 2}(\omega)-\sigma_{1 / 2}(\omega)}{\omega} d \omega
$$

with $\alpha_{e}=1 / 137.04$ and $\sigma_{3 / 2}(\omega)$ and $\sigma_{1 / 2}(\omega)$ the photo-absorption cross sections for parallel and antiparallel nucleon and photon spins, respectively, was determined at two electron accelerators using hydrogen targets. While the measurement from 0.2 to $0.8 \mathrm{GeV}$ was carried out at MAMI [15], the energy range from 0.68 to $2.9 \mathrm{GeV}$ was covered at the electron stretcher ring ELSA [16]. In total, the

Table 3

Measured values of the $\mathrm{GDH}$ integral $I_{\mathrm{GDH}}$ for the proton and model predictions for the unmeasured ranges.

\begin{tabular}{|l|c|c|}
\hline & $E_{\gamma}[\mathrm{GeV}]$ & $I_{\mathrm{GDH}}[\mu b]$ \\
\hline MAID2002 [17] & $0.14-0.20$ & $-27.5 \pm 3$ \\
measured (GDH-Collaboration) & $0.20-2.90$ & $254 \pm 5 \pm 12$ \\
Regge prediction [18] & $>2.9$ & -14 \\
\hline GDH integral & $0.14-\infty$ & $\approx 213$ \\
\hline GDH sum rule & $\nu_{t h r}-\infty$ & 205 \\
\hline
\end{tabular}

range from the resonance region up to the Regge regime was covered. This range is wide enough to reliably make conclusions on the validity of the GDH sum rule for the first time. Only the ranges from $0.14-0.20 \mathrm{GeV}$ and $>2.9 \mathrm{GeV}$ had to be covered using model predictions.

\subsection{Spin polarizability $\gamma_{\pi}$ and $L N$ sum rule}

The LN sum rule [7] is formulated for the backward direction using fixed- $\theta$ dispersion theory. Its $s$-channel part is given by 


$$
\begin{aligned}
\gamma_{\pi}^{s}= & \int_{\omega_{0}}^{\infty} \frac{d \omega}{4 \pi^{2} \omega^{3}} \sqrt{1+\frac{2 \omega}{M}}\left(1+\frac{\omega}{M}\right) \\
& \times \sum_{n} P_{n}\left[\sigma_{3 / 2}^{n}(\omega)-\sigma_{1 / 2}^{n}(\omega)\right]
\end{aligned}
$$

with $P_{n}=+1$ for $M 1, E 2, M 3, \cdots$ multipoles and $P_{n}=-1$ for $E 1, M 2, E 3, \cdots$ multipoles. Its $t$ channel part is given by the contributions of pseudoscalar poles

$$
\begin{aligned}
\gamma_{\pi}^{t}=\frac{1}{2 \pi M}\left[\frac{g_{\pi N N} F_{\pi^{0} \gamma \gamma}}{m_{\pi^{0}}^{2}} \tau_{3}\right. & +\frac{g_{\eta N N} F_{\eta \gamma \gamma}}{m_{\eta}^{2}} \\
& \left.+\frac{g_{\eta^{\prime} N N} F_{\eta^{\prime} \gamma \gamma}}{m_{\eta^{\prime}}^{2}}\right] .
\end{aligned}
$$

The analogous separation in fixed- $t$ dispersion theory into an integral part and an asymptotic (contour integral) part is described in [1]. In the present approach we tentatively make the assumption that $\gamma^{t} \equiv \gamma^{\text {as }}$.

The second line in Table 4 shows the spin po-

Table 4

Experimental results obtained for the backward spinpolarizabilities of proton and neutron compared with predictions from the LN sum rule. Unit of spin polarizability: $10^{-4} \mathrm{fm}^{4}$.

\begin{tabular}{|l|l|l|l|}
\hline spin polarizabil. & proton & neutron & \\
\hline$\gamma_{\pi}($ fixed- $t)$ & $-38.7 \pm 1.8$ & $+58.6 \pm 4.0$ & exp. $[13,19]$ \\
$\gamma_{\pi}($ fixed- $\theta)$ & $-39.5 \pm 2.4$ & $+52.5 \pm 2.4$ & sum rule [7] \\
\hline$\gamma^{t} \equiv \gamma^{\text {as }}$ & -46.6 & +43.4 & $\pi^{0}+\eta+\eta^{\prime}[7]$ \\
$\gamma_{\pi}^{\text {int }}$ & $+7.9 \pm 1.8$ & $+15.2 \pm 4.0$ & exp. [13, 19] \\
$\gamma_{\pi}^{s}$ & $+7.1 \pm 1.8$ & $+9.1 \pm 1.8$ & sum rule [7] \\
\hline
\end{tabular}

larizabilities determined from experimental data $[13,19]$ using fixed- $t$ dispersion theory for the data analysis. Apparently, for the proton the integral part $\gamma^{\text {int }}$ of fixed- $t$ dispersion theory (line 5) and the $s$-channel part $\gamma^{s}$ of fixed- $\theta=\pi$ dispersion theory (line 6 ) are in agreement with each other with good precision. The same is true for the neutron within a larger margin of uncertainty. This leads to the important conclusion that the assumption $\gamma_{\pi}^{t} \equiv \gamma^{\text {as }}$ is confirmed and that the backward spinpolarizability is fully understood.
2.4. The electromagnetic polarizability $(\alpha-\beta)$ and the BEFT sum rule

The $s$-channel part of the BEFT sum rule [6] is given by

$$
\begin{aligned}
(\alpha-\beta)^{s} & =\frac{1}{2 \pi^{2}} \int_{\omega_{0}}^{\infty} \frac{d \omega}{\omega^{2}} \sqrt{1+\frac{2 \omega}{M}} \\
\times & {[\sigma(\Delta P=\text { yes })-\sigma(\Delta P=\text { no })] }
\end{aligned}
$$

where $\Delta P=$ yes for $E 1, M 2, E 3, \cdots$ multipoles and $\Delta P=$ no for $M 1, E 2, E 3, \cdots$ multipoles. The $t$-channel part of the BEFT sum rule is given by

$$
\begin{aligned}
& (\alpha-\beta)^{t}=\frac{1}{16 \pi^{2}} \int_{4 m_{\pi}^{2}}^{\infty} \frac{d t}{t^{2}} \frac{16}{4 M^{2}-t}\left(\frac{t-4 m_{\pi}^{2}}{t}\right)^{1 / 2} \\
& {\left[f_{+}^{0}(t) F_{0}^{0 *}(t)-\left(M^{2}-\frac{t}{4}\right)\left(\frac{t}{4}-m_{\pi}^{2}\right) f_{+}^{2}(t) F_{0}^{2 *}(t)\right],}
\end{aligned}
$$

where $f_{+}^{J}(t)(J=0,2)$ are the amplitudes for the $\pi \pi \rightarrow N \bar{N}$ transition and $F_{0}^{J}(t)$ the amplitudes for the $\gamma \gamma \rightarrow \pi \pi$ transition. Both amplitudes are constructed from the respective imaginary parts through dispersion relations $(N / D$ method) which incorporate the effects of $\pi \pi$ correlations as given by the phase function $\delta_{0}^{J}(t)$ extracted from $\pi \pi$ scattering $(\pi N \rightarrow N \pi \pi)$ data. It is of interest to relate the procedure outlined above to the $\sigma$ meson. The $\sigma$ meson - though strongly demanded by theory as a chiral partner of the $\pi$ meson - has only recently been observed in other experimental data. It has been realized $[20,21]$ that the phase shift $\delta_{0}^{0}(t)$ in scalar $(\mathrm{J}=0)$ and iso-scalar $(\mathrm{I}=0) \pi \pi$ scattering data can consistently be interpreted in terms of a broad resonance if a background is taken into account. This background shifts the position of $\sqrt{s}\left(\delta_{S}=90^{\circ}\right)$ from $\operatorname{Re} \sqrt{s}$ (pole) to about 900 $\mathrm{MeV}$. This leads to the results listed in lines 2 and 3 of Table 5 . Line 5 of Table 5 shows the results of an analysis of the $\gamma \gamma \rightarrow \pi^{0} \pi^{0}$ reaction and line 7 the PDG summary on the pole parameters. Table 6 shows five independent evaluations of the BEFT sum rule, where the last one corresponds to recent unpublished work [28]. The $s$-channel component of this last evaluation $\left(\alpha_{p}-\beta_{p}\right)^{s}=-(5.0 \pm 1.0)$ is in line with all the other results, whereas the $t$-channel result $\left(\alpha_{p}-\beta_{p}\right)=+(14.0 \pm 2.0)$ is in 
Table 5

Position of the $\sigma(600)$ pole, $90^{\circ}$ crossing of the scalar phase and two-photon decay width of the $\sigma$ meson, with supplement a) given by the present author.

\begin{tabular}{|c|c|c|}
\hline$\sqrt{s}($ pole $)[\mathrm{MeV}]$ & $\sqrt{s}\left(\delta_{S}=90^{\circ}\right)$ & method \\
\hline$(470 \pm 30)-i(295 \pm 20)$ & $844 \pm 13 \mathrm{MeV}$ & $\pi N \rightarrow N \pi \pi[20]$ \\
$(585 \pm 20)-i(193 \pm 35)$ & $\sim 900 \mathrm{MeV}$ & $\pi N \rightarrow N \pi \pi[21]$ \\
\hline \hline$\sqrt{s}($ pole $)[\mathrm{MeV}]$ & $\Gamma_{\sigma \rightarrow 2 \gamma}[\mathrm{keV}]$ & \\
\hline$(547 \pm 45)-i(602 \pm 181)$ & $0.62 \pm 0.19$ & $\gamma \gamma \rightarrow \pi^{0} \pi^{0}[22]$ \\
\hline $\mathrm{PDG}$ summary $\left.{ }^{a}\right)$ & & recommended \\
$(500 \pm 40)-i(250 \pm 40)$ & & average [23] \\
\hline
\end{tabular}

a) supplemented by estimated errors.

Table 6

Numerical evaluation of the BEFT sum rule, with corrections a) and b) supplemented by the present author.

\begin{tabular}{|l|l|l|c|}
\hline$\left(\alpha_{p}-\beta_{p}\right)^{s}$ & $\left(\alpha_{p}-\beta_{p}\right)^{t}$ & $\left(\alpha_{p}-\beta_{p}\right)^{\mathrm{BEFT}}$ & authors \\
\hline-4.92 & $+9.28^{a)}$ & +4.36 & {$[24]$} \\
-4 & $+10.4^{b)}$ & +6.4 & {$[25]$} \\
-5.42 & +8.6 & $+\left(3.2_{-3.6}^{+2.4}\right)$ & {$[26]$} \\
-5.56 & +16.46 & $+(10.7 \pm 0.2)^{c)}$ & {$[27]$} \\
\hline$-(5.0 \pm 1.0)$ & $+(14.0 \pm 2.0)$ & $+(9.0 \pm 2.2)$ & {$[28]$} \\
\hline
\end{tabular}

a) correction for the $\mathrm{D}$ wave contribution (-1.7) included. b) correction for the polarizability of the pion $(+3.0)$ included. c) best value from a range of results given by the authors.

agreement with the result of [27] only. Adopting the results of line 6 we arrive at the conclusion that the prediction of the BEFT sum rule, viz. $\left(\alpha_{p}-\beta_{p}\right)^{\mathrm{BEFT}}=+(9.0 \pm 2.2)$, is in line with the experimental value $\left(\alpha_{p}-\beta_{p}\right)^{\exp }=10.1 \pm 0.9$ (see Table 7). This result has to be compared with the expectation that the $\sigma$ meson probably has a twocomponent structure with $|\pi \pi\rangle$ being the one component and $|q \bar{q}\rangle$ being the other [29].

As a summary Table 7 shows the difference between calculated values for $(\alpha-\beta)_{p}$ and the experimental value for this quantity obtained under conditions explained in the caption of the table. We see that in fixed- $t$ dispersion theory we have to explain $(\alpha-\beta)_{p}^{\text {as }}=13.2 \pm 1.3$ through a contribution which has no interpretation in terms of the integral part in fixed- $t$ dispersion theory. This value, therefore, may be interpreted as an empirical result for the asymptotic contribution to $(\alpha-\beta)$. In case of fixed- $\theta$ dispersion theory $(\alpha-\beta)_{p}^{t \text {-miss }}$ is compatible with zero.
Table 7

Predicted information $(\alpha-\beta)_{p}^{\text {calc }}$ on the polarizability difference compared with the experimental result $(\alpha-\beta)_{p}^{\exp }=$ $10.5 \pm 1.1[10]$ or $(\alpha-\beta)_{p}^{\exp }=10.1 \pm 0.9$ (adopted average including all existing data $[9,10])$. In fixed- $t$ dispersion theory the prediction $(\alpha-\beta)_{p}^{\text {calc }}$ corresponds to the integral part $(\alpha-\beta)_{p}^{\mathrm{int}}$, in fixed- $\theta$ dispersion theory $(\alpha-\beta)_{p}^{\text {calc }}$ is either chosen to be the $s$-channel contribution only (line 3 ) or the predicted $s$-channel contribution supplemented by the predicted $\gamma \gamma \rightarrow \pi \pi \rightarrow N \bar{N} t$-channel contribution according to the BEFT sum rule (line 4).

\begin{tabular}{|l|l|l|}
\hline theor. & $(\alpha-\beta)_{p}^{\text {calc }}$ & $(\alpha-\beta)_{p}^{\exp }-(\alpha-\beta)_{p}^{\text {calc }}$ \\
\hline fixed- $t$ & -3.1 (int. part $)[2]$ & $(\alpha-\beta)_{p}^{\text {as }}=13.2 \pm 1.3$ \\
fixed- $\theta$ & $-(5.0 \pm 1.0)(s$-chan. $)$ & $(\alpha-\beta)_{p}^{t \text {-exp }}=15.1 \pm 1.3$ \\
fixed- $\theta$ & $+(9.0 \pm 2.2)(s+t$-ch. $)$ & $(\alpha-\beta)^{t-\text { miss }}=1.1 \pm 2.4$ \\
\hline
\end{tabular}

An interesting alternative for the prediction of $(\alpha-\beta)$ which deserves further consideration has been proposed and evaluated by Akhmedov and Fil'kov [30]. In this approach $(\alpha-\beta)$ is expressed through a dispersion relation at fixed $u=M^{2}$ in the point $t=0$.

\subsection{The effective $\sigma$ pole}

The evaluation of the reaction $\gamma \gamma \rightarrow \pi^{0} \pi^{0}$ [22] has led to a determination of the position of the $\sigma$ pole as well as to the determination of the photon decay width $\Gamma_{\sigma \rightarrow 2 \gamma}$ (Table 5). This makes it interesting to reinvestigate the properties of the effective $\sigma$ pole introduced to approximately represent the asymptotic (contour-integral) contribution [1], using these data as a basis. The relation between $\alpha-\beta$ and the $\sigma$ pole is given by

$$
\frac{g_{\sigma N N} F_{\sigma \gamma \gamma}}{2 \pi m_{\sigma}^{2}}=(\alpha-\beta)^{\sigma-\text { pole }}
$$

where $m_{\sigma}=600 \mathrm{MeV} \hat{=} 3.04 \mathrm{fm}^{-1}$ is the nominal value of the $\sigma$ mass which has been found to be consistent with experimental Compton differential cross-sections in the second resonance region [31]. The relation to the decay width is given by

$$
g_{\sigma N N} F_{\sigma \gamma \gamma}=+16 \pi \sqrt{\frac{g_{\sigma N N}^{2}}{4 \pi} \frac{\Gamma_{\sigma \rightarrow 2 \gamma}}{m_{\sigma}^{3}}}
$$

where the approximate equality of the two coupling constants

$$
\frac{g_{\sigma N N}^{2}}{4 \pi} \simeq \frac{g_{\pi N N}^{2}}{4 \pi}=13.75
$$


may be justified through the linear $\sigma$ model. Using

$$
\Gamma_{\sigma \rightarrow 2 \gamma}=(0.62 \pm 0.19) \mathrm{keV}
$$

evaluated by Fil'kov and Kashevarov [22], we arrive at

$$
(\alpha-\beta)^{\sigma \text {-pole }}=10.7 \pm 1.7 \text {. }
$$

The same calculation may be carried out using the experimental value $m_{\sigma}=(547 \pm 45) \mathrm{MeV}$ as obtained by Fil'kov and Kashevarov [22]. Then we arrive at

$$
(\alpha-\beta)^{\sigma-\text { pole }}=14.8_{-2.5}^{+2.1}\left(\Delta \Gamma_{\sigma \rightarrow 2 \gamma}\right)_{-3.6}^{+5.2}\left(\Delta m_{\sigma}\right) .
$$

It is satisfactory to see that the numbers obtained in (11) and (12) for $(\alpha-\beta)^{\sigma \text {-pole }}$ are in the same range as the number given for $(\alpha-\beta)_{p}^{\text {as }}$ in the third row of Table 7 .

Acknowledgement: The author is indebted to M.I. Levchuk, A.I. L'vov and A.I. Milstein for valuable discussions.

\section{References}

[1] A.I. L'vov, V.A. Petrun'kin, M. Schumacher, Phys. Rev. C 55 (1997) 359-377;

[2] F. Wissmann, SPRINGER TRACTS IN MODERN PHYSICS 200 (2003) 1-132

[3] M. Schumacher, Nucl. Phys. A 721 (2003) 773c-776c; nucl-ex/0309010

[4] A.M. Baldin, Nucl. Phys. 18 (1960) 310-317; L.I. Lapidus, Zh. Eksp. Teor. Fiz. 43 (1962) 1358-1361 [Sov. Phys. JETP 16 (1963) 964-966]

[5] S.B. Gerasimov, Sov. J. Nucl. Phys. 2 (1966) 430-433 [J. Nucl. Phys. (U.S.S.R.) 2 (1965) 598-602]; S.D. Drell, A.C. Hearn, Phys. Rev. Lett. 16 (1966) 908-911

[6] J. Bernabeu, T.E.O. Ericson, C. Ferro Fontan, Phys. Lett. 49 B (1974) 381-384; J. Bernabeu, B. Tarrach, Phys. Lett. 69 B (1977) 484-488

[7] A.I. L'vov, A.M. Nathan, Phys. Rev. C 59 (1999) 10641069

[8] M.I. Levchuk, A.I. L'vov, A.I. Milstein, M. Schumacher, (in preparation)

[9] P.S. Baranov, A.I. L'vov, V.A. Petrun'kin, L.N. Shtarkov, Phys. Part. Nuc. 32 (2001) 376-394

[10] V. Olmos de León et al., Eur. Phys. J. A 10 (2001) $207-215$

[11] M.I. Levchuk, A.I. L'vov, Nucl. Phys. A 674 (2000) 449-492
[12] J. Schmiedmayer et al., Phys. Rev. Lett. 66 (1991) 1015-1018

[13] K. Kossert et al., Phys. Rev. Lett. 88 (2002) 162301-14; Eur. Phys. J. A 16 (2003) 259-273; nucl.-ex/0210020

[14] M. Lundin et al., Phys. Rev. Lett. 90 (2003) 192501$1-4$

[15] J. Ahrens et al., Phys. Rev. Lett. 84 (2000) 5950-5954; Phys. Rev Lett. 87 (2001) 022003-1-5; Phys. Rev. Lett. 88 (2002) 232002-1-5

[16] H. Dutz et al., Phys. Rev. Lett. 91 (2003) 192001-1-5; Phys. Rev. Lett. 93 (2004) 032003-1-5

[17] L. Tiator, Proc. GDH2002, Genova, Italy 3-6 July, World Scientific (2002) 27-36

[18] N. Bianchi, E. Thomas, Phys. Lett. B 450 (1999) 439447; S. Simula et al., Phys. Rev. D 65 (2002) 034017-1-17

[19] M. Camen et al., Phys. Rev. C 65 (2002) 032202-1-3

[20] G. Colangelo, J. Gasser, H. Leutwyler, Nucl. Phys. B 603 (2001) 125-179

[21] M. Ishida, Prog. Theor. Phys. Suppl. 149 (2003) 190202 ; hep-ph/0212383

[22] L.V. Fil'kov, V.L. Kashevarov, Eur. Phys. J. A5 (1999) 285-292

[23] S. Eidelman et al. (Particle Data Group), Phys. Lett. B 592 (2004) 1; URL http://pdg.lbl.gov

[24] I. Guiasu, E.E. Radescu, Phys. Rev. D 18 (1978) 17281730

[25] V.M. Budnev, V.A. Karnakov, Yad. Fiz. 30 (1979) 440-444; [Sov. J. Nucl. Phys. 30 (1979) 228-230]

[26] B.R. Holstein, A.M. Nathan, Phys. Rev. D 49 (1994) 6101-6108

[27] D. Drechsel, B. Pasquini, M. Vanderhaeghen, Phys. Rept. 378 (2003) 99-205

[28] M.I. Levchuk, A.I. L'vov, A.I. Milstein, M. Schumacher, (to be published)

[29] M.D. Scadron, et al., Phys. Rev. D 69 (2004) 014010; Erratum-ibid. D 69 (2004) 059901; hep/0309109

[30] D.M. Akhmedov, L.V. Fil'kov, Sov. J. Nucl. Phys. 33 (1981) 573-578 [Yad. Fiz. 33 (1981) 1083-1095]

[31] G. Galler et al., Phys. Lett. B 503 (2001) 245-255; S. Wolf et al., Eur. Phys. J. A 12 (2001) 231-252 